\title{
SOME STABILITY CONDITIONS FOR A NONLINEAR DIFFERENTIAL EQUATION
}

\author{
BY \\ DON HINTON( $\left.{ }^{1}\right)$
}

1. Introduction. In the following we shall be concerned with the behavior of the solutions of the differential equation

$$
\left(p y^{\prime}\right)^{\prime}+q y^{\gamma}=0,
$$

where $\gamma \geqq 1$ is the ratio of odd, positive integers. The functions $p$ and $q$ are assumed to be real-valued and continuous on a ray $[a, \infty)$. It is further supposed that $p$ and $q$ are positive and have three continuous derivatives.

Problems of particular interest are:

I. When are all solutions of equation (1) bounded on $[a, \infty)$, and when do they all have limit zero?

II. When are all solutions of equation (1) oscillatory on $[a, \infty)$ ?

III. If $y$ is an oscillatory solution of equation (1), can an asymptotic equation be found for the frequency of $y$ ? These three problems are considered in $\$ \S 2,3$, and 4 respectively.

The special case $p(t)=q(t)=t^{2}$ is known as Emden's equation and arises in astrophysics in determining the temperature distribution in polytropic gas spheres [1, p. 528]. The generalization $p(t)=t^{\alpha}$ and $q(t)=t^{\beta}$ of Emden's equation is studied in detail in Chapter 7 of [2].

Classical existence and uniqueness theorems yield solutions of (1), e.g., Theorem 2.3 of Chapter 1 of [3] is applicable. It is also well known that a solution $y$ of (1) can be extended to $[a, \infty)$ if $y$ is bounded on each interval $[a, b)$ on which it exists. That this is the case for all solutions of (1) will be proved in $\S 2$.

2. Boundedness properties. If $y$ is a solution of equation (1), then we define the function $z$ by

$$
z=2(\gamma+1)^{-1} \phi^{-(\gamma+1) / 2} y^{\gamma+1}+\phi\left(p y^{\prime}\right)^{2}
$$

where $\phi=(p q)^{-2 /(\gamma+3)}$. We shall find the function $z$ useful in finding bounds for the growth of $y$ and $p y^{\prime}$. It is to be noted that $y^{\gamma+1}$ is positive if $y$ is negative; hence both terms of the right-hand side of (2) are nonnegative.

LEMMA 1. If $y$ and $z$ are given by (1) and (2) respectively, then

$$
\left[z-\left(p \phi^{\prime}\right) y p y^{\prime}+2^{-1} p\left(p \phi^{\prime}\right)^{\prime} y^{2}\right]^{\prime}=2^{-1}\left[p\left(p \phi^{\prime}\right)^{\prime}\right]^{\prime} y^{2}
$$

Received by the editors January 19, 1968 and, in revised form, May 31, 1968.

( $\left.{ }^{1}\right)$ This research was supported by the National Science Foundation under grant GP-6292. 
Proof. Calculating $z^{\prime}$ from equation (2) and collecting terms gives

$$
z^{\prime}=2\left[\phi^{-(\gamma+1) / 2} y^{\gamma} y^{\prime}+\phi\left(p y^{\prime}\right)\left(p y^{\prime}\right)^{\prime}\right]+\phi^{\prime}\left[-\phi^{-(\gamma+3) / 2} y^{\gamma+1}+\left(p y^{\prime}\right)^{2}\right] .
$$

Since $y$ is a solution of (1), this reduces to

$$
z^{\prime}=\phi^{\prime}\left[-\phi^{-(\gamma+3) / 2} y^{\gamma+1}+\left(p y^{\prime}\right)^{2}\right]
$$

Substitution of this equation into the left-hand side of (3) and a reduction of $\left(p \phi^{\prime}\right) y\left(p y^{\prime}\right)^{\prime}$ by use of (1) proves the lemma.

Lemma 1 for $p \equiv 1$ and $\gamma$ an odd positive integer is used by Wong in [4] to derive conditions that yield all solutions of (1) have limit zero at infinity. Our results in this direction place somewhat different requirements on $p$ and $q$ than those used by Wong.

Our analysis now proceeds in a manner analogous to that used in [5] where the linear equation $\gamma=1$ and $p \equiv 1$ is discussed. We prove that if $p$ and $q$ satisfy certain order relations, then $z$ has a finite limit at infinity.

THEOREM 1. If $y$ and $z$ satisfy equations (1) and (2) respectively and the conditions

(i) $\left|p \phi^{\prime}\right| \rightarrow 0$ anil $\left|p\left(p \phi^{\prime}\right)^{\prime}\right| \phi \rightarrow 0$ as $t \rightarrow \infty$, and

(ii) $\int_{a}^{\infty}\left|\left[p\left(p \phi^{\prime}\right)^{\prime}\right]^{\prime}\right| \phi<\infty$ hold, then there is a number $L \geqq 0$ such that $z(t) \rightarrow L$ as $t \rightarrow \infty$.

Proof. First suppose $z$ is not bounded on $[a, \infty)$. The above conditions imply that there is a number $b$ such that

$$
\int_{b}^{\infty}\left|\left[p\left(p \phi^{\prime}\right)^{\prime}\right]^{\prime}\right| \phi<2^{-1}[2 /(\gamma+1)]^{2 /(\gamma+1)}
$$

and for $t \geqq b$,

$$
\left|p\left(p \phi^{\prime}\right)^{\prime} \phi\right|(t)<2^{-1}[2 /(\gamma+1)]^{2 /(\gamma+1)}
$$

and

$$
\left|p \phi^{\prime}\right|(t)<4^{-1}[2 /(\gamma+1)]^{1 /(\gamma+1)} .
$$

Since $z$ is unbounded there is an increasing sequence $\left\{t_{i}\right\}_{1}^{\infty}$ such that $b \leqq t_{1}, t_{i} \rightarrow \infty$ and $z\left(t_{i}\right) \rightarrow \infty$ as $i \rightarrow \infty, z\left(t_{1}\right)>1$ and

$$
z\left(t_{i}\right)=\max \left\{z(t) \mid b \leqq t \leqq t_{i}\right\} .
$$

Applying Lemma 1 and letting

$$
B=\left|z-\left(p \phi^{\prime}\right) y p y^{\prime}+2^{-1} p\left(p \phi^{\prime}\right)^{\prime} y^{2}\right|(b),
$$

we have the inequality

$$
\left|z-\left(p \phi^{\prime}\right) y p y^{\prime}+2^{-1} p\left(p \phi^{\prime}\right)^{\prime} y^{2}\right|\left(t_{i}\right) \leqq B+2^{-1} \int_{b}^{t_{i}}\left|\left[p\left(p \phi^{\prime}\right)^{\prime}\right]^{\prime}\right| y^{2}
$$

From equation (2) we conclude that

$$
|y| \leqq[(\gamma+1) / 2]^{1 /(\gamma+1)} \phi^{1 / 2} z^{1 /(\gamma+1)}
$$

and

$$
\left|p y^{\prime}\right| \leqq \phi^{-1 / 2} z^{1 / 2}
$$


Inequalities (4), (8) and (9) imply that

$$
\begin{aligned}
2^{-1} \int_{b}^{t_{i}} \mid & {\left[p\left(p \phi^{\prime}\right)^{\prime}\right]^{\prime} \mid y^{2} } \\
& \leqq 2^{-1}[(\gamma+1) / 2]^{2 /(\gamma+1)} \int_{b}^{t_{i}}\left|\left[p\left(p \phi^{\prime}\right)^{\prime}\right]^{\prime}\right| \phi z\left(t_{i}\right)^{2 /(\gamma+1)} \\
& \leqq 4^{-1} z\left(t_{i}\right)^{2 /(\gamma+1)} \leqq 4^{-1} z\left(t_{i}\right)
\end{aligned}
$$

Applying inequalities (8) and (9) and equations (5) and (6), we obtain

$$
2^{-1}\left|p\left(p \phi^{\prime}\right)^{\prime} y^{2}\right|\left(t_{i}\right) \leqq 4^{-1} z\left(t_{i}\right)^{2 /(\gamma+1)} \leqq 4^{-1} z\left(t_{i}\right)
$$

and

$$
\left|\left(p \phi^{\prime}\right)\left(y p y^{\prime}\right)\right|\left(t_{i}\right) \leqq 4^{-1} z\left(t_{i}\right)^{(\gamma+3) / 2(\gamma+1)} \leqq 4^{-1} z\left(t_{i}\right) .
$$

Application of inequalities (10), (11) and (12) to equation (7) yields that

$$
z\left(t_{i}\right)-2^{-1} z\left(t_{i}\right) \leqq B+4^{-1} z\left(t_{i}\right)
$$

or $z\left(t_{i}\right) \leqq 4 B$. This inequality however is a contradiction to $z\left(t_{i}\right) \rightarrow \infty$ as $i \rightarrow \infty$. Thus $z$ is bounded.

Since $z$ is bounded we have from equation (8) that $\phi^{-1} y^{2}$ is bounded and thus $\int_{a}^{\infty}\left|\left[p\left(p \phi^{\prime}\right)^{\prime}\right]^{\prime}\right| y^{2}<\infty$. This implies that the function

$$
r=z-\left(p \phi^{\prime}\right) y p y^{\prime}+2^{-1} p\left(p \phi^{\prime}\right)^{\prime} y^{2}
$$

has a finite limit $L$ at infinity. From (8) and (9) and condition (i) of the theorem it is clear that both $\left(p \dot{\phi}^{\prime}\right) y p y^{\prime}$ and $p\left(p \phi^{\prime}\right)^{\prime} y^{2}$ have limit zero at infinity. Thus $z$ has limit $L$ at infinity, and $L$ is nonnegative since $z$ is positive.

Two corollaries are now immediate.

CoROllary 1.1. Suppose conditions (i) and (ii) of Theorem 1 hold. If for some $c>0, p q \geqq c$, then all solutions of (1) are bounded on $[a, \infty)$. If $(p q) \rightarrow \infty$ as $t \rightarrow \infty$, then all solutions of (1) have limit zero at $\infty$. On the other hand if $p q \leqq c$ for some $c>0$, then $\left(p y^{\prime}\right)$ is bounded on $[a, \infty)$ if $y$ is a solution of $(1)$, and $\left(p y^{\prime}\right) \rightarrow 0$ as $t \rightarrow \infty$ if $(p q) \rightarrow 0$ as $t \rightarrow \infty$.

Corollary 1.2. If conditions (i) and (ii) of Theorem 1 hold and $s>0$, then $\int_{a}^{\infty} \phi^{s / 2}<\infty$ is a sufficient condition that for all solutions $y$ of (1) we have $\int_{a}^{\infty}|y|^{s}<\infty$.

For $p \equiv 1$ and $q(t)=t+3 \sin t$ on [1, $\infty)$, Corollary 1.1 applies, and all solutions of (1) have limit zero at infinity. Considering the equation

$$
\frac{d}{d t}\left(t^{\alpha} \frac{d y}{d t}\right)+t^{\beta} y(t)^{\gamma}=0,
$$

we find conditions (i) and (ii) of Theorem 1 hold if and only if

$$
-2(\alpha+\beta)<(1-\alpha)(\gamma+3) \text {. }
$$


Thus if (15) holds and $2(\alpha+\beta)>(\gamma+3)$, then all solutions of (14) are square integrable on $[1, \infty)$ and have limit zero at infinity.

For our discussion in $\S 4$ we need to have the limit $L>0$. A more immediate result of $L>0$ is that if $y$ in Theorem 1 is oscillatory, then

and

$$
\limsup _{t \rightarrow \infty} y(t)^{2} \geqq[L(\gamma+1) / 2]^{2 /(\gamma+1)} \cdot \liminf _{t \rightarrow \infty} \phi(t)
$$

$$
\limsup _{t \rightarrow \infty}\left(p y^{\prime}\right)(t)^{2} \geqq L \cdot \liminf _{t \rightarrow \infty} \phi(t)^{-1} .
$$

These inequalities follow from equation (2) since at a zero of $y$, the first term on the right-hand side of (2) is zero, and at a zero of $y^{\prime}$ the second term on the right-hand side of (2) is zero. We note that when the above inequalities are satisfied, then $(p q) \rightarrow \infty$ as $t \rightarrow \infty$ implies that the limit superior of $\left|p y^{\prime}\right|$ at infinity is infinite and $(p q) \rightarrow 0$ as $t \rightarrow \infty$ implies that the limit superior of $|y|$ at infinity is infinite. In such cases we cannot have stability in the Lyapunov sense for the identically zero solution of (1).

We now state four theorems which give conditions that imply $L>0$. For each of these theorems we assume $y, z$ and $L$ are as in Theorem 1 and conditions (i) and (ii) are satisfied.

THEOREM 2. If $\gamma=1$, then $L>0$.

Proof. It follows from (8), (9) and (13) that $r / z \rightarrow 1$ as $t \rightarrow \infty$. Now

$$
r^{\prime}=2^{-1}\left[p\left(p \phi^{\prime}\right)^{\prime}\right]^{\prime} y^{2}=\left\{2^{-1}\left[p\left(p \phi^{\prime}\right)^{\prime}\right]^{\prime} y^{2} / r\right\} r
$$

and $y^{2} / r \leqq \phi z / r$. Thus the above equation is of the form $r^{\prime}=A r$ where $\int_{a}^{\infty}|A|<\infty$. Since $r(t)=r(a) \exp \int_{a}^{t} A$, the limit $L$ at infinity is nonzero.

THEOREM 3. If $\left[p\left(p \phi^{\prime}\right)^{\prime}\right]^{\prime} \geqq 0$ and $y$ has a zero, then $L>0$.

Proof. Since $r^{\prime}=2^{-1}\left[p\left(p \phi^{\prime}\right)^{\prime}\right]^{\prime} y^{2}$, we have that $r$ is nondecreasing. At a zero $b$ of $y, r(b)=z(b)>0$. Hence $L>0$.

THEOREM 4. If $\int_{a}^{\infty}\left|(p q)^{\prime}\right|(p q)^{-1}<\infty$, then $L>0$.

Proof. From the calculation of $z^{\prime}$ in Lemma 1, it follows that

$$
z^{\prime} z^{-1}=\phi^{\prime} \phi^{-1}\left[-\phi^{-(\gamma+1) / 2} y^{\gamma+1}+\phi\left(p y^{\prime}\right)^{2}\right] z^{-1} .
$$

This equation implies the inequality

$$
\left|z^{\prime}\right| z^{-1} \leqq\left|\phi^{\prime}\right| \phi^{-1}(\gamma+3) / 2=\left|(p q)^{\prime}\right|(p q)^{-1},
$$

which in turn yields $\int_{a}^{\infty}\left|z^{\prime}\right| z^{-1}<\infty$. This relation implies that $z$ does not have limit zero at infinity.

The inequality $\left|z^{\prime}\right| z^{-1} \leqq\left|(p q)^{\prime}\right|(p q)^{-1}$ implies that

$$
\left|\ln \left[z\left(b^{*}\right) z(a)^{-1}\right]\right| \leqq \int_{a}^{b}\left|(p q)^{\prime}\right|[p q]^{-1}
$$

for $b^{*}<b$. Thus if a solution $y$ of (1) exists on a half-open interval $[a, b)$, it follows that $z$ and hence $y$ are bounded on $[a, b)$. By our earlier remarks, this means every solution of (1) is extendable to $[a, \infty)$. 
THEOREM 5. If $(p q)^{\prime} \geqq 0, g(t)=\phi(t)^{-1} \int_{t}^{\infty}\left|\left[p\left(p \phi^{\prime}\right)^{\prime}\right]^{\prime}\right| \phi, y$ is oscillatory and $g$ is bounded on $[a, \infty)$, then $L>0$.

Proof. A calculation proves that

$$
\left(z \phi^{-1}\right)^{\prime}=-(\gamma+3)(\gamma+1)^{-1} \phi^{-(\gamma+5) / 2} \phi^{\prime} y^{\gamma+1} \geqq 0,
$$

and thus the limit of $z \phi^{-1}$ as $t \rightarrow \infty$ is positive or infinity. Suppose that $L=0$ and the zeros of $y$ are $t_{1}, t_{2}, \ldots$ Then by Lemma 1 ,

$$
\begin{aligned}
z\left(t_{i}\right) & =-2^{-1} \int_{t_{i}}^{\infty}\left[p\left(p \phi^{\prime}\right)^{\prime}\right]^{\prime} y^{2} \\
& \leqq 2^{-1}[(\gamma+1) / 2]^{2 /(\gamma+1)} \int_{t_{i}}^{\infty}\left|\left[p\left(p \phi^{\prime}\right)^{\prime}\right]^{\prime}\right| \phi z^{2 /(\gamma+1)} .
\end{aligned}
$$

After multiplying both sides of equation (16) by $\phi\left(t_{i}\right)^{-1}$ we find that the limit as $i \rightarrow \infty$ of the right-hand side of (16) is zero. This however implies that $z\left(t_{i}\right) \phi\left(t_{i}\right)^{-1}$ $\rightarrow 0$ as $i \rightarrow \infty$ which is a contradiction.

Returning to equation (14) we see by Theorems 2, 3 and 5 respectively, that $L>0$ if (15) is satisfied and one of the following conditions: (i) $\gamma=1$, (ii) $y$ has a zero and $\alpha+\beta \leqq 0$ and (iii) $y$ is oscillatory and $\alpha+\beta \geqq 0$.

3. Conditions for oscillation. Some of our previous theorems required that a solution $y$ of (1) be oscillatory. For $\gamma=1$, effective conditions for detecting oscillation are given in [6]. We concern ourselves here with the nonlinear case.

THEOREM 6. If $\gamma>1$, then a necessary and sufficient condition that all nontrivial solutions of (1) be oscillatory is that

$$
\int_{a}^{\infty}\left(\int_{a}^{t} p(v)^{-1} d v\right) q(t) d t=\infty \quad \text { if } \int_{a}^{\infty} p(v)^{-1} d v=\infty
$$

and

$$
\int_{a}^{\infty}\left(\int_{t}^{\infty} p(v)^{-1} d v\right)^{\gamma} q(t) d t=\infty \quad \text { if } \int_{a}^{\infty} p(v)^{-1} d v<\infty
$$

Proof. This result is a corollary of a result of Atkinson's [7, Theorem 1] which states that all solutions of $y^{\prime \prime}+q y^{\gamma}=0$ are oscillatory if and only if $\int_{a}^{\infty} t q(t) d t=\infty$. Actually, Atkinson states his theorem for $\gamma$ an odd positive integer; the more general case is given by Licko and Švec [8] whose results are also applicable to higher order equations.

For the case $\int_{a}^{\infty} p(v)^{-1} d v=\infty$, let $h(t)=\int_{a}^{t} 1 / p$ and $g$ be the inverse of $h$, i.e., $g(h(t))=t$ for all $t \geqq a$. Let $y$ be a solution of (1) and define $w(s)=y(g(s))$ for $s \geqq 0$. It follows readily that $w$ satisfies the differential equation

$$
w^{\prime \prime}(s)+Q(s) w(s)^{\gamma}=0
$$

where $Q(s)=[p q](g(s))$. Conversely, if $w$ is a solution of $(19)$ and $y(t)=w(h(t))$, then $y$ is a solution of (1). Clearly, $y$ is oscillatory if and only if $w$ is, and Atkinson's 
result states that $w$ is oscillatory if and only if $\int_{0}^{\infty} s Q(s) d s=\infty$. The change of variable $s=h(t)$ proves that this condition is equivalent to (17).

For the case $\int_{a}^{\infty} p(v)^{-1} d v<\infty$, let $h(t)=\left(\int_{t}^{\infty} 1 / p\right)^{-1}$ and $g$ be the inverse of $h$. For a solution $y$ of (1) we define $w$ by $w(s)=s y(g(s))$. The function $w$ is then a solution of $w^{\prime \prime}(s)+Q(s) w(s)^{\gamma}=0$ where $Q(s)=s^{-(\gamma+3)}[p q](g(s))$. The proof now proceeds as above and follows from the relation

$$
\int_{h(a)}^{\infty} s Q(s) d s=\int_{a}^{\infty}\left(\int_{t}^{\infty} p(v)^{-1} d v\right)^{\gamma} q(t) d t
$$

Returning to (14), we find for $\gamma>1$ that conditions (17) and (18) are respectively $\alpha-\beta \leqq 2$ if $\alpha \leqq 1$ and $\beta+(1-\alpha) \gamma \geqq-1$ if $\alpha>1$.

We mention also that Kurzweil [9] has given a sufficient condition that the equation $y^{\prime \prime}+q y^{2 n-1}=0$ have an oscillatory solution, and Atkinson [7, Theorem 2] states a sufficient condition that no solution of $y^{\prime \prime}+q y^{2 n-1}=0$ be oscillatory. Surprisingly, more effective criteria for the detection of an oscillatory solution seems to be available for higher-order equations. A result of J. W. Heidel [10] states that if $n \geqq 3$ is odd and $\gamma>1$, then the equation $y^{(n)}+q y^{\gamma}=0$ has an oscillatory solution if $\int_{a}^{\infty} x^{n-2+\gamma} q(x) d x=\infty$.

4. Asymptotic distribution of zeros. The following lemma will be useful in the sequel.

LEMMA 2. Suppose $f$ is a continuously differentiable positive function on $[a, \infty)$ and for some $\mu \geqq 0,\left|f^{\prime}(t)\right| f(t)^{-\mu} \rightarrow 0$ as $t \rightarrow \infty$. If $\varepsilon$ and $K$ are positive numbers, then there is a number $B$ such that if $t$ and $s$ are $\geqq B$ and $|t-s| \leqq K f(s)^{1-\mu}$, then

$$
\left|f(t) f(s)^{-1}-1\right|<\varepsilon
$$

Proof. Choose $B$ such that $t \geqq B$ implies that

$$
\left|f^{\prime}(t)\right| f(t)^{-\mu} \leqq \varepsilon / 2 K(1+\varepsilon)^{\mu} .
$$

Let $s \geqq B$ and define $g(t)=f(t) f(s)^{-1}$ for all $t$ such that $t \geqq B$ and $|t-s| \leqq K f(s)^{1-\mu}$. Since $g(s)=1$, if $|g(t)-1| \geqq \varepsilon$ for some $t$, then there is a number $t^{*}$ such that $\left|g\left(t^{*}\right)-1\right|=\varepsilon$ and $|g(t)-1|<\varepsilon$ for all $t$ between $s$ and $t^{*}$. In such case we then have for some $t$ between $s$ and $t^{*}$ that

$$
\begin{aligned}
\varepsilon & =\left|g\left(t^{*}\right)-1\right|=\left|g^{\prime}(t)\right|\left|t^{*}-s\right| \\
& =\left|f^{\prime}(t)\right| f(s)^{-1}\left|t^{*}-s\right| \leqq f(t)^{\mu} f(s)^{-1} \varepsilon\left|t^{*}-s\right| / 2 K(1+\varepsilon)^{\mu} .
\end{aligned}
$$

Since $\left|t^{*}-s\right| \leqq K f(s)^{1-\mu}$, the above inequality reduces to

$$
\varepsilon \leqq f(t)^{\mu} f(s)^{-\mu} \varepsilon / 2(1+\varepsilon)^{\mu}=\varepsilon g(t)^{\mu} / 2(1+\varepsilon)^{\mu} .
$$

Hence $(1+\varepsilon)^{\mu}<2(1+\varepsilon)^{\mu} \leqq g(t)^{\mu}$. Now $|g(t)-1|<\varepsilon$ implies that $g(t)^{\mu} \leqq(1+\varepsilon)^{\mu}$, contrary to the above inequality. Hence $B$ chosen as above establishes the lemma.

We shall now prove a theorem which describes the asymptotic behavior of the zeros of an oscillatory solution of (1). 
THEOREM 7. Suppose $p \equiv 1, y$ is an oscillatory solution of (1) with zeros $t_{1}, t_{2}, \ldots$ and intermediate zeros of $y^{\prime}, t_{1}^{\prime}, t_{2}^{\prime}, \ldots, z$ is given by (2) and $z(t) \rightarrow L>0$ as $t \rightarrow \infty$. If $\left|\phi^{\prime}(t)\right| \rightarrow 0$ as $t \rightarrow \infty$, then

$$
\left[t_{i}^{\prime}-t_{i}\right] q\left(t_{i}^{\prime}\right)^{2 /(\gamma+3)} \rightarrow \Pi_{\gamma} C \text { as } i \rightarrow \infty
$$

and

$$
\left[t_{i+1}-t_{i}^{\prime}\right] q\left(t_{i}^{\prime}\right)^{2 /(\gamma+3)} \rightarrow \Pi_{\gamma} C \quad \text { as } i \rightarrow \infty
$$

where

$$
\Pi_{\gamma}=\int_{0}^{1}\left[1-u^{\gamma+1}\right]^{-1 / 2} d u \text { and } C=[L(1+\gamma) / 2]^{(1-\gamma) / 2(1+\gamma)}[(1+\gamma) / 2]^{1 / 2} .
$$

Proof. Since $\phi=q^{-2 /(\gamma+3)}$, the condition $\left|\phi^{\prime}(t)\right| \rightarrow 0$ as $t \rightarrow \infty$ is equivalent to $\left|q^{\prime}(t)\right| q(t)^{-(\gamma+5) /(\gamma+3)} \rightarrow 0$ as $t \rightarrow \infty$. We first derive an inequality for $t_{i}^{\prime}-t_{i}$ with the aid of Lemma 2. Let

$$
K=2(\gamma+1)(2 L)^{1 / 2} /[L(1+\gamma) / 4]^{\gamma /(1+\gamma)}
$$

and consider $y$ on $\left[t_{i}, t_{i}^{\prime}\right]$. For $t \in\left[t_{i}, t_{i}^{\prime}\right)$ such that

$$
\left|t-t_{i}^{\prime}\right| \leqq K q\left(t_{i}^{\prime}\right)^{-2 /(\gamma+3)}
$$

we have by integrating (1),

$$
y^{\prime}(t)=\int_{t}^{t_{i}^{i}} q y^{\nu}
$$

Since $y$ is concave on $\left[t_{i}, t_{i+1}\right],|y(s)| \geqq\left|y\left(t_{i}^{\prime}\right)\right|(s-t)\left(t_{i}^{\prime}-t\right)^{-1}$ for $t \leqq s \leqq t_{i}^{\prime}$; hence it follows from (23) that

$$
\begin{aligned}
\left|y^{\prime}(t)\right| & \geqq \int_{t}^{t_{i}^{\prime}} q(s)\left[\left|y\left(t_{i}^{\prime}\right)\right|(s-t)\left(t_{i}^{\prime}-t\right)^{-1}\right]^{\gamma} d s \\
& \geqq q_{t}\left|y\left(t_{i}^{\prime}\right)\right|^{\gamma}\left(t_{i}^{\prime}-t\right) /(\gamma+1)
\end{aligned}
$$

where $q_{t}$ is the minimum of $q$ on $\left[t, t_{i}^{\prime}\right]$. Since $\phi(t) y^{\prime}(t)^{2} \leqq z(t)$ and

$$
z\left(t_{i}^{\prime}\right)=2(\gamma+1)^{-1} \phi\left(t_{i}^{\prime}\right)^{-(\gamma+1) / 2} y\left(t_{i}^{\prime}\right)^{\gamma+1},
$$

the above inequality reduces to

$$
\left(t_{i}^{\prime}-t\right) \leqq \frac{(\gamma+1) z(t)^{1 / 2} \phi(t)^{-1 / 2} \phi\left(t_{i}^{\prime}\right)^{-\gamma / 2}}{q_{t}\left[z\left(t_{i}^{\prime}\right)(1+\gamma) / 2\right]^{\gamma /(1+\gamma)}} .
$$

It is sufficient to consider $i$ sufficiently large so that

$$
\frac{(\gamma+1) z(t)^{1 / 2}}{\left[z\left(t_{i}^{\prime}\right)(1+\gamma) / 2\right]^{\gamma /(1+\gamma)}} \leqq \frac{(\gamma+1)(2 L)^{1 / 2}}{[L(1+\gamma) / 4]^{\gamma /(1+\gamma)}}=K / 2 ;
$$

hence (24) reduces to

$$
\left(t_{i}^{\prime}-t\right) \leqq \frac{K}{2 q\left(t_{i}^{\prime}\right)^{2 /(\gamma+3)}}\left[\frac{q\left(t_{i}^{\prime}\right)}{q_{t}}\right]\left[\frac{q(t)}{q\left(t_{i}^{\prime}\right)}\right]^{1 /(\gamma+3)}
$$


Applying Lemma 2 for $f=q$ and $\mu=(\gamma+5) /(\gamma+3)$, we may consider $i$ sufficiently large so that

$$
\left[q_{t} / q\left(t_{i}^{\prime}\right)\right]^{-1}\left[q(t) / q\left(t_{i}^{\prime}\right)\right]^{1 /(\gamma+3)} \leqq 3 / 2
$$

in which case $(25)$ reduces to

$$
\left(t_{i}^{\prime}-t\right) \leqq 3 K / 4 q\left(t_{i}^{\prime}\right)^{2 /(\gamma+3)} .
$$

Since (26) holds for those $t \in\left[t_{i}, t_{i}^{\prime}\right]$ satisfying (22), we conclude that (22) holds for $t=t_{i}$ and all sufficiently large $i$.

With the aid of (22) we now prove (20). For $t \in\left[t_{i}, t_{i}^{\prime}\right]$, it follows after an integration of $y^{\prime \prime} y^{\prime}=-q y^{\gamma} y^{\prime}$ that

$$
y^{\prime}(t)^{2}=2 \int_{t}^{t_{i}^{\prime}} q y^{\gamma} y^{\prime}
$$

Denote by $q_{i}$ and $Q_{i}$ the minimum and maximum respectively of $q$ on $\left[t_{i}, t_{i}^{\prime}\right]$. From (27) we then have that

$$
2 q_{i}\left[y\left(t_{i}^{\prime}\right)^{\gamma+1}-y(t)^{\gamma+1}\right] /(\gamma+1) \leqq y^{\prime}(t)^{2} \leqq 2 Q_{i}\left[y\left(t_{i}^{\prime}\right)^{\gamma+1}-y(t)^{\gamma+1}\right] /(\gamma+1)
$$

and thus

$$
\begin{aligned}
{\left[2 q_{i} y\left(t_{i}^{\prime}\right)^{\gamma+1} /(\gamma+1)\right]^{1 / 2} } & \leqq\left|y^{\prime}(t)\right|\left[1-\left(y(t) / y\left(t_{i}^{\prime}\right)\right)^{\gamma+1}\right]^{-1 / 2} \\
& \leqq\left[2 Q_{i} y\left(t_{i}^{\prime}\right)^{\gamma+1} /(\gamma+1)\right]^{1 / 2}
\end{aligned}
$$

Integrating this inequality over $\left[t_{i}, t_{i}^{\prime}\right]$, it follows that

$$
\begin{aligned}
{\left[2 q_{i} y\left(t_{i}^{\prime}\right)^{\gamma+1} /(\gamma+1)\right]^{1 / 2}\left(t_{i}^{\prime}-t_{i}\right) } & \leqq\left|y\left(t_{i}^{\prime}\right)\right| \Pi_{\gamma} \\
& \leqq\left[2 Q_{i} y\left(t_{i}^{\prime}\right)^{\gamma+1} /(\gamma+1)\right]^{1 / 2}\left(t_{i}^{\prime}-t_{i}\right) .
\end{aligned}
$$

Since $\left|y\left(t_{i}^{\prime}\right)\right|=\left[(\gamma+1) z\left(t_{i}^{\prime}\right) / 2\right]^{1 /(\gamma+1)} q\left(t_{i}^{\prime}\right)^{-1 /(\gamma+3)}$,

$$
\begin{aligned}
{\left[2 q_{i} /(\gamma+1)\right]^{1 / 2}\left|y\left(t_{i}^{\prime}\right)\right|^{(\gamma-1) / 2}=} & {[2 /(\gamma+1)]^{1 / 2}\left[q_{i} / q\left(t_{i}^{\prime}\right)\right]^{1 / 2} } \\
& \cdot\left[(\gamma+1) z\left(t_{i}^{\prime}\right) / 2\right]^{(\gamma-1) / 2(\gamma+1)} q\left(t_{i}^{\prime}\right)^{2 /(\gamma+3)} .
\end{aligned}
$$

By Lemma $2, q_{i} / q\left(t_{i}^{\prime}\right) \rightarrow 1$ as $i \rightarrow \infty$; hence applying (29) to the left-hand side of (28) yields

$$
\limsup _{i \rightarrow \infty} q\left(t_{i}^{\prime}\right)^{2 /(\gamma+3)}\left(t_{i}^{\prime}-t_{i}\right) \leqq \Pi_{\gamma} C .
$$

Similar considerations yield the opposite inequality for the limit inferior of $q\left(t_{i}^{\prime}\right)^{2 /(\gamma+3)}\left(t_{i}^{\prime}-t_{i}\right)$. These two inequalities imply Equation (20). Equation (21) is derived in an analogous manner.

As a consequence of this theorem and Lemma 2, we have that if $s_{1}, s_{2}, \ldots$ is a sequence such that $t_{i} \leqq s_{i} \leqq t_{i+1}$ for each $i$, then $q\left(s_{i}\right) / q\left(t_{i}^{\prime}\right) \rightarrow 1$ as $i \rightarrow \infty$. Thus equations (20) and (21) remain valid if $q\left(t_{i}^{\prime}\right)$ is replaced by $q\left(s_{i}\right)$.

Another conclusion that is an immediate consequence of Theorem 7 is that the zeros of $y^{\prime}$ are spaced approximately halfway between the zeros of $y$ in the sense that $\left(t_{i+1}-t_{i}^{\prime}\right)\left(t_{i}^{\prime}-t_{i}\right)^{-1} \rightarrow 1$ as $i \rightarrow \infty$. 
The asymptotic result given by Bellman [2, p. 163] for the distance between zeros of solutions of $u^{\prime \prime}+t^{\sigma} u=0$ follows readily by combining equations (20) and (21) to obtain an asymptotic expression for $t_{i+1}-t_{i}$. The formula given by Kiguradze $\left[11\right.$, p. 136] for the distance between zeros of solutions of $u^{\prime \prime}+a u^{\gamma}=0$ with $a^{\prime} \geqq 0$ is obtained by combining equations (20) and (21) and replacing $t_{i}^{\prime}$ by $t_{i}$.

We can use Theorem 7 to solve the more general problem where $p \not \equiv 1$.

THEOREM 8. Suppose $y, z, t_{i}, t_{i}^{\prime}$ and $L$ are as in Theorem 7 and $p$ satisfies the conditions: (i) $\int_{a}^{\infty} 1 / p=\infty$, (ii) $\left|p \phi^{\prime}\right| \rightarrow 0$ as $t \rightarrow \infty$ and (iii) for some $\mu \geqq 0, p^{\mu} \phi$ is bounded on $[a, \infty)$ and $\left|p^{\prime}\right| p^{-\mu} \rightarrow 0$ as $t \rightarrow \infty$. Then

$$
\left(t_{i}^{\prime}-t_{i}\right)\left[p\left(t_{i}^{\prime}\right) \phi\left(t_{i}^{\prime}\right)\right]^{-1} \rightarrow \Pi_{\gamma} C \quad \text { as } i \rightarrow \infty
$$

and

$$
\left(t_{i+1}-t_{i}^{\prime}\right)\left[p\left(t_{i}^{\prime}\right) \phi\left(t_{i}^{\prime}\right)\right]^{-1} \rightarrow \Pi_{\gamma} C \quad \text { as } i \rightarrow \infty .
$$

Proof. Let $h, g^{\prime}, w$ and $Q$ be defined as in the first half of the proof of Theorem 6 . Choose $t_{i}^{*}$ such that $\int_{t_{i}}^{t_{i}^{\prime}} 1 / p=\left(t_{i}^{\prime}-t_{i}\right) / p\left(t_{i}^{*}\right)$ and let $s_{i}=h\left(t_{i}\right), s_{i}^{\prime}=h\left(t_{i}^{\prime}\right)$ and $s_{i}^{*}=h\left(t_{i}^{*}\right)$. Then the zeros of $w$ are $s_{1}, s_{2}, \ldots$ and the zeros of $w^{\prime}$ are $s_{1}^{\prime}, s_{2}^{\prime}, \ldots$ For

$$
\Phi(s)=Q(s)^{-2 /(\gamma+3)}=\phi(g(s)),
$$

we have $\Phi^{\prime}(s)=\left(p \phi^{\prime}\right)(g(s))$; hence $\left|\Phi^{\prime}(s)\right| \rightarrow 0$ as $s \rightarrow \infty$. For

$$
Z(s)=2(\gamma+1)^{-1} \Phi(s)^{-(\gamma+1) / 2} w(s)^{\gamma+1}+\Phi(s) w^{\prime}(s)^{2}=z(g(s)),
$$

it follows that $Z(s) \rightarrow L$ as $s \rightarrow \infty$ and thus Theorem 7 applies to $w$. Applying Theorem 7 and the remarks following the proof of Theorem 7, we conclude that if $s_{i} \leqq s_{i}^{\prime \prime} \leqq s_{i+1}$ for each $i$, then

$$
\left(s_{i}^{\prime}-s_{i}\right) Q\left(s_{i}^{\prime \prime}\right)^{2 /(\gamma+3)} \rightarrow \Pi_{\gamma} C \quad \text { as } i \rightarrow \infty .
$$

Since $s_{i}^{\prime}-s_{i}=\int_{t_{i}}^{t_{i}^{\prime}} 1 / p=\left(t_{i}^{\prime}-t_{i}\right) / p\left(t_{i}^{*}\right)$, the above limit is for $s_{i}^{\prime \prime}=s_{i}^{*}$,

$$
\left(t_{i}^{\prime}-t_{i}\right)\left[p\left(t_{i}^{*}\right) \phi\left(t_{i}^{*}\right)\right]^{-1} \rightarrow \Pi_{\gamma} C \quad \text { as } i \rightarrow \infty .
$$

Since $p^{\mu} \phi$ is bounded there is a number $M$ such that

$$
\left(t_{i}^{\prime}-t_{i}\right) p\left(t_{i}^{*}\right)^{\mu-1}=\left(t_{i}^{\prime}-t_{i}\right)\left[p\left(t_{i}^{*}\right) \phi\left(t_{i}^{*}\right)\right]^{-1} p\left(t_{i}^{*}\right)^{\mu} \phi\left(t_{i}^{*}\right) \leqq M
$$

for all $i$. From Lemma 2 for $f=p$ the limit $p\left(t_{i}^{\prime}\right) / p\left(t_{i}^{*}\right) \rightarrow 1$ as $i \rightarrow \infty$ is obtained. Using this limit and (32) for $s_{i}^{\prime \prime}=s_{i}^{\prime}$ yields the equation (30). Equation (31) is derived by similar considerations.

THEOREM 9. Suppose that $y, z, t_{i}, t_{i}^{\prime}, L$ and $p$ are as in Theorem 8 . Let $N(b)$ be the number of zeros of $y$ in $[a, b]$. Then

$$
N(b)^{-1}\left[\int_{a}^{b}(p \phi)^{-1}\right] \rightarrow 2 \Pi_{\gamma} C \quad \text { as } b \rightarrow \infty .
$$


Proof. Choose $t_{i}^{\prime \prime}$ for each $i$ so that

$$
\int_{t_{i}}^{t_{i}^{\prime}}(p \phi)^{-1}=\left(t_{i}^{\prime}-t_{i}\right)\left[p\left(t_{i}^{\prime \prime}\right) \phi\left(t_{i}^{\prime \prime}\right)\right]^{-1}
$$

Employing now the limit (32) for $s_{i}^{\prime \prime}=h\left(t_{i}^{\prime \prime}\right)$ yields

$$
\left(t_{i}^{\prime}-t_{i}\right)\left[p\left(t_{i}^{*}\right) \phi\left(t_{i}^{\prime \prime}\right)\right]^{-1} \rightarrow \Pi_{\gamma} C \quad \text { as } i \rightarrow \infty \text {. }
$$

In the same manner that we proved $p\left(t_{i}^{\prime}\right) / p\left(t_{i}^{*}\right) \rightarrow 1$ as $i \rightarrow \infty$ in Theorem 8 , it follows that $p\left(t_{i}^{\prime \prime}\right) / p\left(t_{i}^{*}\right) \rightarrow 1$ as $i \rightarrow \infty$. This remark, together with above equations implies that $\int_{t_{i}}^{t_{i}^{\prime}}(p \phi)^{-1} \rightarrow \Pi_{\gamma} C$ as $i \rightarrow \infty$. Similarly we have

and these limits yield

$$
\int_{t_{i}^{i}}^{t_{i+1}}(p \phi)^{-1} \rightarrow \Pi_{\gamma} C \quad \text { as } i \rightarrow \infty
$$

$$
\int_{t_{l}}^{t_{i+1}}[p \phi]^{-1} \rightarrow 2 \Pi_{\gamma} C \text { as } i \rightarrow \infty .
$$

The limit (33) implies that the arithmetic means have the same limit, i.e.,

$$
i^{-1} \int_{t_{1}}^{t_{i+1}}(p \phi)^{-1} \rightarrow 2 \Pi_{\gamma} C \quad \text { as } i \rightarrow \infty
$$

For $t_{i} \leqq b<t_{i+1}, N(b)=i$. Thus the theorem is an immediate consequence of (34).

In the linear case $\gamma=1, C=1$ and $\Pi_{\gamma}=\Pi / 2$. Thus the limits in Theorems 8 and 9 are independent of $L$. In particular the conclusion of Theorem 9 becomes

$$
N(b)^{-1} \int_{a}^{b}(q / p)^{1 / 2} \rightarrow \Pi \quad \text { as } b \rightarrow \infty .
$$

\section{REFERENCES}

1. V. A. Ambartsumyan, Theoretical astrophysics, Pergamon Press, New York, 1958.

2. Richard Bellman, Stability theory of differential equations, McGraw-Hill, New York, 1953.

3. E. A. Coddington and N. Levinson, Theory of ordinary differential equations, McGrawHill, New York, 1955.

4. J. S. W. Wong, Some stability conditions for $x^{\prime \prime}+a(t) x^{2 n-1}=0$, SIAM J. Appl. Math. 15 (1967), 889-892.

5. D. B. Hinton, Some stability conditions for $y^{\prime \prime}+q y=0$, J. Math. Anal. Appl. 21 (1968), 126-131.

6. Zeev Nehari, Oscillation criteria for second-order linear differential equations, Trans. Amer. Math. Soc. 85 (1957), 428-445.

7. F. V. Atkinson, On second-order non-linear oscillations, Pacific J. Math. 5 (1955), 643-647.

8. Imrick Licko and Marko Švec, Le charactere oscillatione des solutions de l'equation $y^{(n)}+f(x) y^{\alpha}=0, n>1$, Czecholovak Math. J. (88) 13 (1963), 481-491.

9. Jaroslav Kurzweil, $A$ note on oscillatory solutions of the equation $y^{\prime \prime}+f(x) y^{2 n-1}=0$, Casopis Pěst. Mat. 85 (1960), 357-358.

10. J. W. Heidel, The existence of oscillatory solutions for a nonlinear odd order differential equation, (submitted to J. Math. Anal. Appl.).

11. I. T. Kiguradze, On the asymptotic properties of solutions of the equation $u^{\prime \prime}+a(t) u^{n}=0$, Soobšč. Akad. Nauk Gruzin. SSR 30 (1963), 129-136.

UNIVERSITY OF GEORGIA, Athens, Georgia 